\title{
Incidence and characteristics of health care-associated infection in hospitalized patients with rheumatic diseases in Alexandria Main University Hospital Abeer A. Abdelatia ${ }^{\mathrm{a}}$ Eman A. Sultan ${ }^{\mathrm{b}}$
}

aDepartment of Internal Medicine, Faculty of Medicine, ${ }^{b}$ Department of Community Medicine, Faculty of Medicine, Alexandria University, Egypt

Correspondence to Abeer Ali Abdelati, MD, Sidi Gaber Street, Alexandria, 21523, Egypt. Tel: 002034265910;

e-mail: abiraliabir@yahoo.com

Received 17 June 2018

Accepted 14 August 2018

Egyptian Rheumatology \& Rehabilitation 2018, 45:148-152

\begin{abstract}
Introduction
Health care-associated infection (HCAI) is an important cause of morbidity and mortality in patients with autoimmune diseases. Most previous reports investigated $\mathrm{HCAl}$ in the setting of systemic lupus erythematosus but lacked reports on other connective tissue diseases.

Objectives

We aimed to determine the incidence and characteristics of HCAI in patients with rheumatic diseases admitted to the Rheumatology Unit in Alexandria Main University Hospital in 2017.

Patients and methods

A 1-year duration prospective observational study was done to elucidate the incidence and characteristics of HCAl in patients with underlying rheumatic diseases who were admitted to our Rheumatology Unit between January 1 and December 31, 2017. Statistical analysis was performed using IBM SPSS Statistics 20. Data are presented as numbers and percentages for categorical variables and means and SDs for continuous variables.

\section{Results}

A total of 516 patients [423 (82\%) female and $93(18 \%)$ male] with underlying rheumatic diseases and disease duration of $6.03 \pm 4.18$ years were admitted during the study period. The mean age was $32.18 \pm 9.54$ years, and the mean length of hospitalization was $10.04 \pm 5.76$ days. HCAl occurred in $14.9 \%(n=77)$ of patients, with $81(15.69 \%)$ infection cases (four cases had more than one episode of infection in more than one site with a different organism during the same admission). Gramnegative bacilli were the most commonly isolated organisms $(46.3 \% ; n=38)$, and the urinary tract was the most commonly documented site of infection $(39.5 \% ; n=32)$. Klebsiella spp. (17.9\%; $n=15)$ was the most frequently identified infectious agent. Of the total cases with $\mathrm{HCAI}(n=77), 85.7 \%(n=66)$ were cured, $7.8 \%(n=6)$ deteriorated, $5.2 \%(n=4)$ died, and $1.3 \%(n=1)$ referred to another specialized hospital.

\section{Conclusion}

Despite the improvement in health care services, the incidence of HCAl infection in our inpatient population is still high and represents a burden on our resources. Although most cases were treated with appropriate antimicrobials, HCAl is still the cause of deterioration, and death occurred in a considerable percentage of patients with rheumatic diseases.
\end{abstract}

\section{Keywords:}

health care-associated infection, incidence, rheumatic diseases

Egypt Rheumatol Rehabil 45:148-152

(c) 2018 Egyptian Society for Rheumatology and Rehabilitation

$1110-161 \mathrm{X}$

\section{Introduction}

Despite a significant increase in the survival of patients with rheumatic disorders over the past five decades, infection continues to be a major cause of mortality and morbidity among those patients, and deaths owing to infection are observed all through the disease course [1]. The increased susceptibility to infections in such patients may be explained by abnormalities of the cellular and humoral immune responses, the use of immunosuppressive drugs, as well as comorbidities [2,3].

Health care-associated infections (HCAI) are an important cause of morbidity and mortality during hospitalization. The estimated number of HCAI cases in hospitals in the USA was 1.7 million, with almost 99000 deaths directly resulting from HCAIs in a study conducted in 2002 [4]

Rapid medical, surgical, and pharmaceutical advances in the past 30 years have resulted in a rapid growth of a population of patients with suppressed immune

This is an open access journal, and articles are distributed under the terms of the Creative Commons Attribution-NonCommercial-ShareAlike 4.0 License, which allows others to remix, tweak, and build upon the work non-commercially, as long as appropriate credit is given and the new creations are licensed under the identical terms. 
function. The incidence of infections in this group has risen rapidly as their numbers grow due to increased survival. Overall, the incidence of serious infections and severe sepsis has clearly increased over time [5].

Infections are an important cause of morbidity in patients with autoimmune diseases [6]. Infections in patients with underlying autoimmune diseases can involve unusual organisms requiring atypical pharmacological therapy. In addition to having increased susceptibility to common communityacquired and nosocomial pathogens, immunocompromised patients are vulnerable to opportunistic pathogens and to reactivation of endogenous but latent organisms [7]. Infection with these pathogens can present with minimal signs and symptoms or with atypical features in unusual locations. This can considerably delay the diagnosis if the presence of immunocompromise is not appreciated. Although the risk of mortality is high in these patients given the underlying immunosuppression and the unusual nature of the involved organisms, the outcome can be optimized by early diagnosis and aggressive treatments with specific therapy $[8,9]$.

It is extremely important to prevent HCAI in medical care generally and in the setting of rheumatic diseases particularly owing to the immunosuppressive state associated with these disorders. There are few detailed systematic reports on HCAI in rheumatology locally and internationally; most previous reports investigated infection as a whole in rheumatic patients or HCAI in the setting of systemic lupus erythematosus (SLE) but lack other connective tissue diseases (CTDs), and reviewing the literatures revealed few studies in this context.

We aimed to determine and characterize the annual incidence of HCAIs in patients with underlying rheumatic diseases admitted to the Rheumatology Unit of the Internal Medicine Department in Alexandria Main University Hospital (AMUH).

\section{Patients and methods}

A 1-year duration prospective observational study was performed to elucidate the incidence and characteristics of HCAIs in patients with underlying rheumatic diseases admitted to the rheumatology ward of the Internal Medicine Department in AMUH, between January 1 and December 31, 2017. Informed consent was obtained from patients who participated in this study and confidentiality of data was assured. Data were collected on demographic and clinical characteristics and also on previous and current clinical history. Infection acquired at any time before admission or detected within $48 \mathrm{~h}$ of hospital admission in patients without previous contact with health care services was classified as community-acquired infection $[10,11]$. HCAI was determined according to the criteria proposed by Cardoso et al. [12] as infection detected within $48 \mathrm{~h}$ of hospital admission in patients who had previous contact with health care service within 1 year. Patients who fulfilled these criteria were counted as patients with HCAI, whereas other patients who did not fulfill these criteria were counted in the non-HCAI group. The diagnosis of infection was based on clinical findings, positive cultures, gram-stain results, specific serological assays, and/or response to antimicrobial treatment. The type of identified organisms, site, and outcome of all HCAI were recorded for each case.

\section{Statistical analysis}

Statistical analysis was performed using IBM SPSS Statistics 20 (Armonk, NY: IBM Corp.) [13]. Data are presented as numbers and percentages for categorical variables and means and SD for continuous variables (age, length of stay, and disease duration). Regarding ethical considerations, official approvals for the study were obtained from the ethical committee of the Faculty of Medicine, University of Alexandria, and the Director of the AMUH. Confidentiality of data was assured.

\section{Results}

During the aforementioned period, a total of 516 admissions were recorded. Their demographic data and clinical characteristics are summarized in Table 1. In $14.9 \%(n=77)$ of the total admissions, at least one HCAI was documented (Fig. 1). Five cases showed mixed infection with more than one type of organism (total $n=82$ ), seven developed a mixed infection with more than one microorganism isolated from the same site (total $n=84$ ), and four patients developed more than one infection in more than one site per admission (total $n=81$ ). Gramnegative bacilli were the most commonly isolated organism in $46.3 \%(n=38)$ followed by gram-positive cocci in $25.6 \%(n=21)$ and fungal infection in $11 \%$ $(n=9)$. Gram-positive diplococci, gram-negative cocci, and gram-negative diplococci were identified in (6.1\%; $n=5),(4.9 ; n=4)$, and $(2.5 \% ; n=2)$, respectively. Grampositive bacilli, gram-negative coccobacilli, and viral infection were documented in $1.2 \%(n=1)$ for each one of them (Table 2). 
Table 1 Demographic and clinical characteristics of the studied cases

\begin{tabular}{lc}
\hline Demographic and clinical characteristics & $n(\%)$ \\
\hline Sex & \\
Male & $93(18.0)$ \\
Female & $423(82.0)$ \\
Place of admission & \\
Male Medical Ward & $73(14.2)$ \\
Female Medical Ward & $336(65.1)$ \\
Intermediate Care Unit & $107(20.7)$ \\
Age (years) & \\
Mean \pm SD & $32.18 \pm 9.54$ \\
Minimum-maximum & $15-54$ \\
Length of stay (days) & \\
Mean \pm SD & $10.04 \pm 5.76$ \\
Minimum-maximum & $4-67$ \\
Diagnosis & \\
Systemic lupus erythematosus & $191(37.0)$ \\
Rheumatoid arthritis & $170(32.9)$ \\
Seronegative spondyloarthritis & $32(6.2)$ \\
Primary vasculitis & $28(5.4)$ \\
Inflammatory myositis & $21(4.1)$ \\
Juvenile idiopathic arthritis & $20(3.9)$ \\
Behçet's disease & $11(2.1)$ \\
Primary antiphospholipid syndrome & $10(1.9)$ \\
Systemic sclerosis & $9(1.7)$ \\
Adult-onset still's disease & $7(1.4)$ \\
Overlap syndrome & $7(1.4)$ \\
Mixed connective tissue disease & $5(1.0)$ \\
Primary Sjogren's syndrome & $5(1.0)$ \\
Disease duration (years) & \\
Mean $\pm S D$ & $6.03 \pm 4.18$ \\
Minimum-maximum & $1-27$ \\
Disease activity & \\
Yes & \\
No & \\
Yes & \\
No & \\
Total & \\
\hline
\end{tabular}

Klebsiella spp. was the most commonly isolated organism in $17.9 \%(n=15)$ followed by methicillinresistant Staphylococcus aureus (MRSA) in 14.3\% $(n=12)$ and Pseudomonas aeruginosa in $11.9 \%(n=10)$, whereas, Pneumocystis jiroveci, Clostridium difficile, Enterovirus spp., and Aspergillus spp. were the least isolated organisms in $1.2 \%(n=1)$ of HCAI cases, for each one of them (Table 3 ).

Urinary tract infection (UTI) was the most common identified infection in $39.5 \% \quad(n=32)$ followed by blood stream infection (BSI) in $18.5 \%$ $(n=15)$, lower respiratory tract infection in $16.1 \%$ $(n=13)$, and upper respiratory tract infection in $7.4 \%$ $(n=6)$, whereas pseudomembranous colitis, infective endocarditis, oropharyngeal infection, and empyema were the least documented infection in only
Figure 1

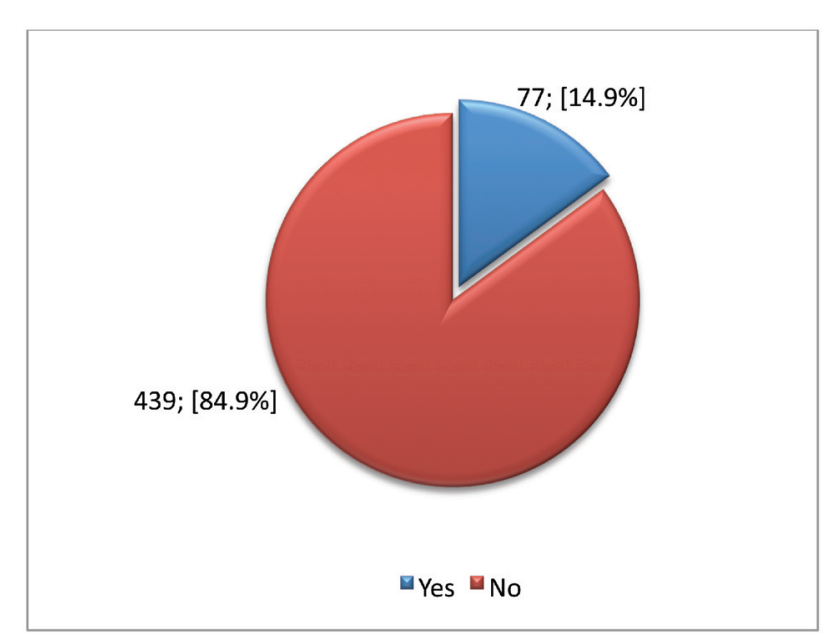

Distribution of studied cases according to the presence of HCAl. HCAl, health care-associated infection.

Table 2 Types of organisms in cases with health careassociated infection

\begin{tabular}{lc}
\hline Type of organisms & $n(\%)$ \\
\hline Gram-negative bacilli & $38(46.3)$ \\
Gram-positive cocci & $21(25.6)$ \\
Fungus & $9(11.0)$ \\
Gram-positive diplococci & $5(6.1)$ \\
Gram-negative cocci & $4(4.9)$ \\
Gram-negative diplococci & $2(2.5)$ \\
Gram-positive bacilli & $1(1.2)$ \\
Gram-negative coccobacilli & $1(1.2)$ \\
Viral $_{\text {Total }}{ }^{\text {a }}$ & $1(1.2)$ \\
\hline
\end{tabular}

${ }^{a}$ Five cases had mixed infection with more than one type of organisms.

1.2\% $(n=1)$ of HCAI cases for each one of them (Table 4).

Regarding the outcome of HCAI cases, $85.7 \%(n=66)$ improved, $7.8 \%(n=6)$ died on the same admission, $5.2 \%(n=4)$ deteriorated and developed multiorgan failure and were shifted to the ICU, and one case with meningitis was transferred to another specialized center for further management (Fig. 2).

\section{Discussion}

Patients with rheumatic diseases have an increased risk of infections owing to their condition and immunosuppressive therapies. To date, reviewing the literature did not reveal any documented data regarding the incidence of overall infection in rheumatic diseases, and HCAI in particular, in Egypt. To decrease the incidence rate of HCAIs, we prospectively surveyed the incidence and characteristics of HCAI in our hospital. 
Table 3 Organisms in cases with health care-associated infection

\begin{tabular}{lc}
\hline Organisms & $n(\%)$ \\
\hline Klebsiella spp. & $15(17.9)$ \\
MRSA & $12(14.3)$ \\
Pseudomonas aeruginosa & $10(11.9)$ \\
Candida albicans & $7(8.3)$ \\
Acinetobacter baumannii & $6(7.1)$ \\
Staphylococcus aureus & $5(5.9)$ \\
Enterococcus species & $5(5.9)$ \\
Escherichia coli & $4(4.7)$ \\
Staphylococcus epidermidis & $3(3.6)$ \\
Legionella pneumophila & $3(3.6)$ \\
Vancomycin-resistant Enterococcus spp. & $2(2.4)$ \\
Providencia spp. & $2(2.4)$ \\
Neisseria meningitidis & $2(2.4)$ \\
Morganella morganii & $2(2.4)$ \\
Chlamydia trachomatis & $2(2.4)$ \\
Pneumocystis jiroveci & $1(1.2)$ \\
Clostridium difficile & $1(1.2)$ \\
Enterovirus spp. & $1(1.2)$ \\
Aspergillus spp. & $1(1.2)$ \\
Total &
\end{tabular}

a Seven cases had mixed infection with more than one organism. MRSA, methicillin-resistant Staphylococcus aureus.

Table 4 Distribution of cases with health care-associated infection according to site of infection

\begin{tabular}{lc}
\hline Site of infections & $n(\%)$ \\
\hline Urinary tract infection & $32(39.5)$ \\
Blood stream infection & $15(18.5)$ \\
Lower respiratory tract infection & $12(14.8)$ \\
Upper respiratory tract infection & $6(7.4)$ \\
Decubitus ulcer infection & $4(5.0)$ \\
Meningitis & $3(3.7)$ \\
Vasculitic ulcer infection & $2(2.5)$ \\
Gastrointestinal tract infection & $2(2.5)$ \\
Septic bursitis & $1(1.2)$ \\
Pseudomembranous colitis & $1(1.2)$ \\
Infective endocarditis & $1(1.2)$ \\
Oropharyngeal infection & $1(1.2)$ \\
Empyema $_{\text {Total }}{ }^{\mathrm{a}}$ & $1(1.2)$ \\
\hline
\end{tabular}

${ }^{a}$ Four cases had infection in more than one site.

HCAI occurred in $14.9 \%$ of patients per year in our study period. UTI was the most common infection in $39.5 \%$ followed by BSI in $18.5 \%$ and RTI in $23.5 \%$ (lower respiratory tract infection in $16.1 \%$ and upper respiratory tract infection in 7.4\%). In a similar study, Masahiro et al. [14], prospectively investigated the incidence of HCAI in a rheumatology care university hospital. They reported an incidence rate of $18.3 \%$ per year in their study period. In their study, the incidence rate of RTI was the highest, and MRSA was the causative organism in $21 \%$ of cases. In contrast to our result, UTI was reported in only $1.0 \%$ of HCAI

\section{Figure 2}

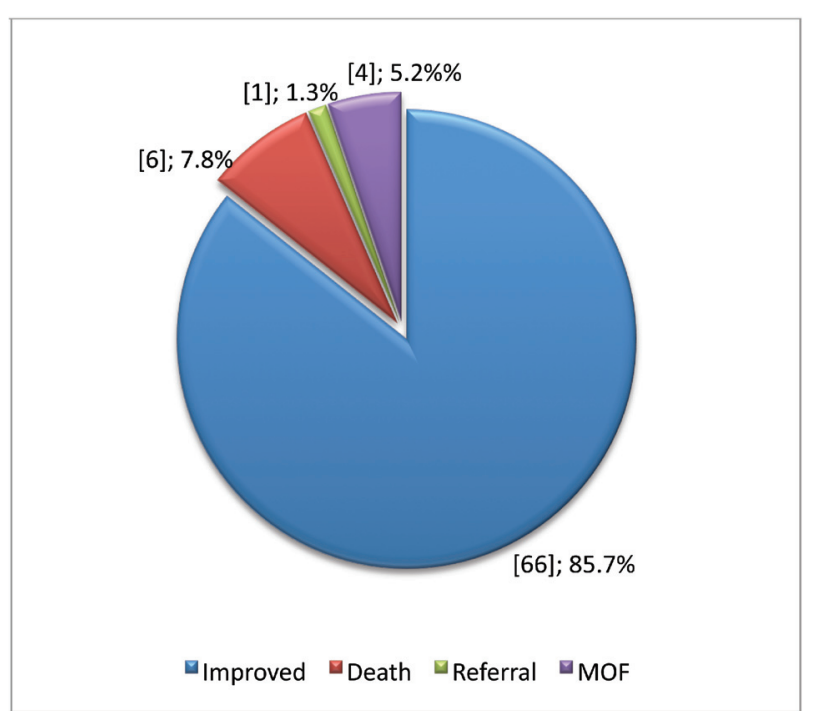

Distribution of cases with $\mathrm{HCAl}$ according to their outcome. $\mathrm{HCAl}$, health care-associated infection; MOF, multiorgan failure.

cases, and they concluded that RTI, BSI, and opportunistic infection were the most common causes of HCAI in patients with CTDs.

Vieira et al. [15] investigated the prevalence of overall infections in their inpatient population at rheumatology unit, and they reported hospitalacquired infection in $19.0 \%$, whereas infections were the admission reason in $17.6 \%$ of the cases, with an overall of $31.7 \%$ period prevalence of infection. In concordance with our results, the urinary tract was the most commonly affected site (32.0\%). However, Escherichia coli was the most frequently identified infectious agent in their study (17.5\%), whereas in the present study, Klebsiella spp. was the most commonly isolated organism in $17.9 \%$ followed by MRSA in $14.3 \%$ of cases with HCAI.

In another two studies, Li et al. [16] and Duffy et al. [17] reported that the major sites of HCAI were the urinary tract, the blood stream, and the respiratory tract. These findings were consistent with our findings. Moreover, in retrospective studies on patients with SLE, HCAI was reported to occur in $\sim 10$ patients per year [18]. However, there are some differences between their studies and our study regarding study designs (retrospective vs. prospective) or study patients (SLE vs. patients with all rheumatic diseases).Previous studies of infection among patients with rheumatic diseases revealed that patients with CTD and infection do poorly compared with those who do not have an infection and that infections contribute to death both early and late in the course of the disease. In 
a study conducted by Abu-Shakra et al. [19], infection was the primary cause of death in $32 \%$ of a cohort of 665 patients with SLE. In the present study, although 85.7\% of HCAI cases had improved on antimicrobial therapy with complete elimination of infection, 7.8\% died from infection on the same admission, whereas $5.2 \%$ deteriorated and developed multiorgan failure and were shifted to the ICU, and one case with meningitis was transferred to another specialized center for further management.

Falagas et al. [20] conducted a systematic review on 39 studies focusing on infection-related morbidity and mortality in patients with CTD; however, most reviewed studies focused on infection incidence and/ or outcome in patients with SLE, and none of the studies focused on HCAI in particular. The mortality attributed to infection was $5.2 \%$. They concluded that infections were a major cause of mortality in patients with CTD.

\section{Conclusion}

In conclusion, identifying the characteristics of HCAI in our patient population will guide us, in the future, in applying preventive measures, anticipation of the nature of infection and proper selection of empirical antimicrobial therapy for early management.

However, the incidence of HCAI in our hospital, site of infections, as well as the causative microorganisms were comparable to the results reported previously in different studies conducted in specialized centers for rheumatology care. UTI, BSI, and RTI were the most common causes of HCAI in our hospital. Despite the improvement in health care services, the incidence of HCAI infection in our inpatient population is still high and represents a burden on our resources, and contributes to the morbidity and mortality in patients with rheumatic diseases.

\section{Financial support and sponsorship Nil.}

\section{Conflicts of interest}

There are no conflicts of interest.

\section{References}

1 Listing J, Gerhold K, Zink A. The risk of infections associated with rheumatoid arthritis, with its comorbidity and treatment. Rheumatology (Oxford) 2013; 52:53-61.

2 Galloway JB, Hyrich KL, Mercer LK, Dixon WG, Fu B, Ustianowski AP, et al. Anti-TNF therapy is associated with an increased risk of serious infections in patients with rheumatoid arthritis especially in the first 6 months of treatment: updated results from the British Society for Rheumatology Biologics Register with special emphasis on risks in the elderly. Rheumatology 2011; 50:124-131.

3 Crawford M, Curtis JR. Tumor necrosis factor inhibitors and infection complications. Curr Rheumatol Rep 2008; 10:383-389.

4 McFee RB. Nosocomial or hospital-acquired infections: an overview. Dis Mon 2009; 55:422-438.

5 Martin GS, Mannino DM, Eaton S, Moss M. The epidemiology of sepsis in the United States from1979 through 2000. N Engl J Med 2003; 348:1546-1554.

6 Doria A, laccarino L, Ghirardello A, Zampieri S, Arienti S, Sarzi-Puttini P, et al. Long-term prognosis and causes of death in systemic lupus erythematosus. Am J Med 2006; 119:700-706.

7 Kuhen BM. Growing list of infections linked to TNF blockers. JAMA 2011; 306:1430.

8 Coello R, Charlett A, Ward V, Wilson V, Wilson J, Pearson A, et al. Devicerelated sources of bacteraemia in English hospitals-opportunities for the prevention of hospital-acquired bacteraemia. J Hosp Infect 2003; 53:46-57.

9 Singh JA, Cameron C, Noorbaloochi S, Cullis T, Tucker M, Christensen R, et al. Risk of serious infection in biological treatment of patients with rheumatoid arthritis: a systematic review and meta-analysis. Lancet 2015; 18:258-265.

10 Friedman ND, Kaye KS, Stout JE, McGarry SA, Trivette SL, Briggs JP, et al. Healthcare-associated bloodstream infections in adults: a reason to change the accepted definition of community-acquired infections. Ann Intern Med 2002; 137:791-797.

11 Siegman-Igra $Y$, Fourier B, Orni-Wasserlauf R, Golan $Y$, Noy A, Schwartz $\mathrm{D}$, et al. Reappraisal of community-acquired bacteremia: a proposal of a new classification for the spectrum of acquisition of bacteremia. Clin Infect Dis 2002; 34:1431-1439.

12 Cardoso T, Almeida M, Friedman ND, Aragao I, Costa-Pereira A, Sarmento $A E$, et al. Classification of healthcare-associated infection: a systematic review 10 years after the first proposal. BMC Med 2014; 12:40.

13 IBM Corp. IBM SPSS statistics for Windows, version 20.0. Armonk, NY: IBM Corp; 2011

14 Masahiro I, Takeshi K, Takao N, Yasuyuki K, Yoko A, Sachiko O, et al. Healthcare-associated infections in rheumatology in Japan. Rheumatol Int 2012; 32:801-804.

15 Vieira R, Fonseca R, Pinto JA, Costa L. Infections in an inpatient rheumatology unit: how big is the problem? Acta Reumatol Port 2016; 41:380-381.

16 Li Z, Chen L, Tao R, Fan X. Clinical and bacteriologic study of eighty-six patients with systemic lupus erythematosus complicated by infections. Chin Med J 1998; 111:913-991.

17 Duffy KN, Duffy CM, Gladman DD. Infection and disease activity in systemic lupus erythematosus: a review of hospitalized patients. J Rheumatol 1991; 18:1180-1184.

18 Navarro-Zarza JE, Alvarez-Hernández E, Casasola-Vargas JC, EstradaCastro E, Burgos-Vargas R. Prevalence of community-acquired and nosocomial infections in hospitalized patients with systemic lupus erythematosus. Lupus 2010; 19:43-48.

19 Abu-Shakra M, Urowitz MB, Gladman DD, Gough J. Mortality studies in systemic lupus erythematosus. Results from a single center. Causes of death. J Rheumatol 1995; 22:1259-1264.

20 Falagas ME, Manta KG, Betsi GI, Pappas G. Infection-related morbidity and mortality in patients with connective tissue diseases: a systematic review. Clin Rheumatol 2007; 26:663-670. 